\title{
Nourriture (Préhistoire) : alimentation carnée
}

Souhila Merzoug

\section{CpenEdition}

Journals

Édition électronique

URL : https://journals.openedition.org/encyclopedieberbere/2760

DOI : 10.4000/encyclopedieberbere. 2760

ISSN : 2262-7197

\section{Éditeur}

Peeters Publishers

\section{Édition imprimée}

Date de publication : 5 octobre 2012

Pagination : 5622-5625

ISBN : 978-90-429-2718-6

ISSN : 1015-7344

\section{Référence électronique}

Souhila Merzoug, « Nourriture (Préhistoire) : alimentation carnée », Encyclopédie berbère [En ligne], 34 2012, document N70, mis en ligne le 15 décembre 2020, consulté le 17 février 2022. URL : http:// journals.openedition.org/encyclopedieberbere/2760 ; DOI : https://doi.org/10.4000/ encyclopedieberbere. 2760

Ce document a été généré automatiquement le 17 février 2022.

(c) Tous droits réservés 


\title{
Nourriture (Préhistoire) : alimentation carnée
}

\author{
Souhila Merzoug
}

1 On traitera ici de l'alimentation préhistorique des derniers chasseurs cueilleurs telliens et atlasiques d'Algérie qui évoquent le mieux des comportements en voie de mutation, passant de chasses ou récoltes opportunistes à des chasses spécialisées, organisées qui révèlent une maîtrise de l'éthologie de certaines espèces.

2 L'alimentation carnée est obtenue par le biais de la chasse et/ou du charognage d'animaux sauvages, ou bien par l'abattage de têtes de troupeaux domestiques. Durant les temps préhistoriques, la chasse, opportuniste ou spécialisée, constituait l'une des activités fondamentales des sociétés humaines, caractérisées par un système économique fondé sur l'exploitation du monde vivant sauvage. La pêche, la cueillette de végétaux et la collecte de mollusques complétaient le système. On parle de sociétés de chasseurs-cueilleurs, par opposition aux sociétés agro-pastorales qui apparaissent au cours de la "Révolution Néolithique " avec l'avènement d'une économie de production. On a longtemps pensé que la pratique de la chasse constituait une évolution dans les comportements de subsistance par rapport au charognage réservé aux Hominiens archaïques. Cependant, grâce aux analyses archéozoologiques des assemblages osseux de sites préhistoriques, il est actuellement admis que les sociétés dites de chasseurs-cueilleurs pratiquaient aussi, de manière opportuniste, le charognage.

3 Au Maghreb, les analyses archéozoologiques sont peu nombreuses et les informations concernant les stratégies d'acquisition et les modes de traitement des animaux abattus restent fragmentaires. Toutefois, grâce à certains travaux récents, on commence à mieux les cerner.

Durant le Paléolithique inférieur et moyen, il est difficile de distinguer la pratique de la chasse et du charognage. Les deux sont suggérées et probablement pratiquées. Les chasseurs préhistoriques du Paléolithique inférieur se nourrissaient essentiellement de viande d'équidés. D'autres espèces ont également été consommées tels que les grands bovinés, les antilopes ainsi que des espèces de très grande taille comme le rhinocéros et 
l'hippopotame (Sahnouni 1998). Au cours du Paléolithique moyen, les Préhistoriques semblent cibler et orienter leurs choix vers un type de gibier en particulier. En effet, dans les sites marocains de la région de Rabat-Témara (El-Harhoura 1 et 2 et ElMnasra; Aouraghe 2001, Campmas ed al. 2008), les populations préhistoriques privilégiaient un gibier de petite taille, les gazelles en l'occurrence étaient très fréquentes (environs 56 individus à El-Harhoura 1). Toutefois, d'autres espèces figuraient également au menu comme les grandes antilopes et les équidés. En Algérie, dans le gisement algérois des Allobroges, c'est un gibier de grande taille qui fut recherché : le buffle antique (12 individus) et l'aurochs (22 individus), constituaient une part importante de l'apport carné (Hadjouis 1994).

5 Au cours du Paléolithique supérieur final, les Hommes de Mechta Afalou de la culture Ibéromaurusienne, pratiquaient une chasse spécialisée, orientée vars un gibier localement abondant. En effet, ces chasseurs constituaient des populations territorialisées, ils puisaient leur subsistance quotidienne dans des chasses locales. Dans la région montagneuse des Babors (Est algérien), le gibier dominant est le mouflon à manchettes* (Ammotragus lervia). Les analyses archéozoologiques effectuées sur les restes osseux des sites de Tamar Hat et Taza 1 ont démontré que ces chasseurs ramenaient en grotte des carcasses entières de mouflons à manchettes. On estime qu'à Taza 1, environ 48 individus ont été chassés et consommés durant 2300 ans d'occupations interrompues. A Tamar Hat, ce sont quelques 174 individus qui ont été dénombrés pour une période d'occupation d'environ 6500 ans. Ces estimations générales n'ont qu'une valeur indicative, très approximative, puisque les remplissages archéologiques n'ont pas été totalement fouillés. Il est aussi difficile d'établir une estimation du nombre d'individus consommés durant les différentes périodes d'occupations saisonnières de ces sites. D'autant plus que d'autres paramètres entrent en compte tels que la durée des occupations, le nombre des habitants, sans oublier le taux de conservation et de fossilisation des ossements.

6 Pour ce qui est du mode de traitement culinaire des mouflons à manchette, il a été établi que les chasseurs de l'Ibéromaurusien découpaient les carcasses avant de faire cuire les parties anatomiques séparément. Aucune trace de carcasses entières cuites n'a été constatée. Toutes les parties du mouflon à manchette ont été consommées, mais les plus recherchées étaient, comme pour le mouton, le squelette axial (notamment les côtelettes) et les membres inférieurs (notamment le gigot). Ces chasseurs poussaient l'exploitation de ce gibier jusqu'à la récupération de la moelle osseuse. La consommation de la cervelle est également attestée.

7 Dans la région des Hautes Plaines du Sersou (Ouest algérien), le gibier recherché était l'alcélaphe (Alcelaphus buselaphus). Au moins 25 individus ont été abattus dans le site de Columnata durant l'Ibéromaurusien. Toutefois, il est difficile de savoir à quoi ce nombre se rapporte en termes d'occupations saisonnières. Le mode d'exploitation culinaire est semblable à celui du mouflon à manchettes.

8 En Libye, selon la région concernée, la sélection d'un gibier particulier est opérée : le mouflon à manchette à Haua Fteah et la gazelle dans la région de Hagfet-Et-Tera. Une exploitation poussée du gibier est encore une fois soulignée (Klein et Scott 1986).

Durant l'Epipaléolithique, une nouvelle population apparaît, les Capsiens* dont les sites conservent de grandes quantités de coquilles de gastéropodes, ce qui leur a valu le surnom de "mangeurs d'escargots». Leur alimentation carnée est essentiellement tirée de l'alcélaphe, des grands bovinés (buffle antique et aurochs) et des gazelles. Le 
lièvre semble, par ailleurs, avoir joué un rôle important dans l'économie de subsistance des Capsiens. L'apport de carcasses entières d'alcélaphe et la présence d'individus jeunes, voire très jeunes dans le site de Dra Mta El-Ma El-Abiod, firent supposer à Morel $(1974,1979,1981)$ la pratique d'un parcage de cet animal, voire même, un début de domestication. Mais, des chercheurs ayant étudié les faunes d'autres sites capsiens tels que Medjez II*, Ain Mestehyia et Kef Zohra penchent plutôt pour la pratique de chasse sélective de ce gibier (Camps-Fabrer 1975, Lubell et al. 1975, 1982-3, 2008).

\section{BIBLIOGRAPHIE}

AOURAGHE H., 2001 - Contribution à la connaissance des faunes pléistocènes supérieures du Maroc : les vertébrés d'El Harhoura (Témara) comparés à ceux de plusieurs sites du Maghreb, Thèse de Doctorat d'Etat, Université Mohamed $1^{\text {er }}$, Oujda, 543 p.

CAMPMAS E., Michel P., AMANI F., COCHARD D., COSTAMAGNO S., NESPOULET R., EL HAJRAOUI M.A., 2008 «Comportements de subsistance à l'Atérien et au Néolithique au Maroc Atlantique : Premiers résultats de l'étude taphonomique et archéozoologique des faunes d'El Harhoura 2 (Région de Témara, Maroc) », in AOURAGHE H., HADDOUMI H., EL HAMMOUTI K. (Eds.), Le Quaternaire marocain dans son contexte méditerranéen. Actes de la Quatrième Rencontre des Quaternaristes Marocains (RQM4), 15-17 novembre 2007, Oujda, p. 236-254.

CAMPS G. \& MOREL J., 1983 - « Recherches sur l'alimentation en Afrique du Nord durant les temps épipaléolithiques ", Bulletin de la Société d'Études et de Recherches Préhistoriques, 33, p. 37-49.

CAMPS-FABRER H., 1975 - Un gisement capsien de faciès sétifien : Medjez II, El-Eulma (Algérie), Paris, Édition du CNRS.

HADJOUIS D., 1994 - « Taphonomie des faunes du gisement atérien des Phacochères (Alger, Algérie) », Artefact 9, p. 183-191.

JACKES M. \& LUBELL D., 2008 - «Environmental and cultural change in the early and mid Holocene : evidence from the Télidjène Basin, Algeria", African Archaeological Review, 25 (1-2), p. 41-55.

KLEIN R.G. \& SCOTT K., 1986 - "Re-analyse of faunal assemblage from the Haua Fteah and other late quaternary archaeological sites in Cyrenaican Libya", Journal of Archaeological Science, 13, p. 515-542.

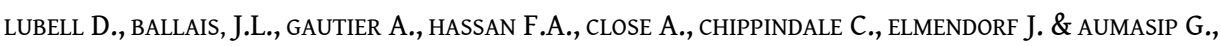
1975 - "Prehistoric culture ecology of Capsian escargotières, I : preliminary results of an interdisciplinary investigation in the Chéria-Télidjène region 1972-73”, Libyca, 23, p. 43-121. LUBELL D., GAUTIER A., LEVENTAL E.T., THOMPSON M., SCHWARCZ H.P. \& SKINNER M., 1982-1983 - “The prehistoric culture ecology of Capsian escargotières, II : Report on investigations conducted during 1976 in the Bahiert Télidjène, Tebessa Wilaya, Algeria", Libyca, 30-31, p. 59-143. 
MERZOUG S., 2005 - Comportements de subsistance des Ibéromaurusiens d'après l'analyse archéozoologique des mammiferes des sites de Tamar Hat, Taza 1 et Columnata (Algérie), Thèse de Doctorat, Muséum National d'Histoire Naturelle, Paris, $412 \mathrm{p}$.

MERZOUG S., 2008 - «Étude archéozoologique des mammifères ibéromaurusiens de la collection Cadenat du site de Columnata (Tiaret, Algérie) », in AOURAGHE, H., HADDOUMI, H., EL HAMMOUTI, K. (Eds.), Le Quaternaire marocain dans son contexte méditerranéen. Actes de la Quatrième Rencontre des Quaternaristes Marocains (RQM4), 15-17 novembre 2007, Oujda, p. 255-264.

MERZOUG S. \& SARI L., 2008 - "Re-examination of the Zone I material from Tamar Hat (Algeria) : Zooarchaeological and technofunctional analyses”, African Archaeological Review, 25, p. 57-73.

MOREL J., 1974 - « La faune de l'escargotière de Dra Mta El-Ma El-Abiod (sud algérien) », L'Anthropologie, 78 (2), p. 299-320.

MOREL J., 1978 - « Les sources d'alimentation des épipaléolithiques de Tamar Hat et le problème des origines de la domestication en Afrique du Nord ", Bulletin du Musée d'Anthropologie Préhistorique de Monaco, 22, p. 73-78.

MOREL J., 1979 - « Sur certains aspects de la vie des populations capsiennes (Épipaléolithique nord-africain) », Bulletin du Musée d'Anthropologie Préhistorique de Monaco, 23, p. 89-102.

MOREL J., 1981 - « Nouvelles réflexions sur l'alimentation et le mode de vie des Capsiens de Dra Mta El-Ma El-Abiod dans la région de Tébessa (Est algérien) », in ROUBET C., HUGOT H.-J., SOUVILLE G. (eds.), Préhistoire africaine : Mélanges offerts au Doyen Lionel Balout, Paris, Editions ADPF, p. 189-194. SAHNOUNI M., 1998 - The lower Palaeolithic of the Maghreb : Excavation and analysis at Aïn Hanech, Algeria. Cambridge monographs in African Archaeology 42.

\section{INDEX}

Mots-clés : Alimentation, Préhistoire 